\title{
Malnutrition as a Complication of Bariatric Surgery - A Clear and Present Danger?
}

\author{
Jessica Lange Alfred Königsrainer \\ Department of General, Visceral and Transplantation Surgery, University Hospital Tübingen, Tübingen, Germany
}

Keywords

Malnutrition · Nutritional status · Obesity · Postoperative monitoring $\cdot$ Sleeve gastrectomy

\section{Abstract}

Obesity is a chronic life-threatening disease, and bariatric surgery is the most effective treatment in those patients. The two main operations are laparoscopic sleeve gastrectomy (LSG) and Roux-en-Y gastric bypass (RYGB). LSG carries a smaller risk for nutritional deficiencies, while gastric bypass procedures are associated with increased nutritional deficiencies because the procedure is more complex and changes the gastrointestinal anatomy. Recent studies comparing LSG and RYGB have proven that these types of operation may lead to a similar weight reduction effect but cause different micronutrient deficiencies. Types of malnutrition after bariatric surgery include protein-energy malnutrition and deficiencies of micronutrients, such as iron, folate, vitamin A, and vitamin $B_{12}$. Bariatric patients who do not adhere to the recommended diets are at a greater risk of developing relevant malnutrition. Therefore, life-long postoperative clinical and laboratory monitoring is necessary to diagnose deficiencies of vitamins, trace elements, and minerals and to correct them with supplements. Unfortunately, no standardized aftercare regimes exist for these patients, and the costs for nutritional supplements are paid by the patients themselves.

(c) 2019 S. Karger AG, Basel

\section{Introduction}

According to the World Health Organization (WHO), at least 2.8 million people die every year because of being overweight or obese. The worldwide prevalence of obesity increased between 1980 and 2014, with 13\% of the world's adult population found to be obese in a survey conducted in 2014 [1]. Obesity is associated with increased morbidity and mortality. Therapeutic options are based on diet, physical activity, behavioral therapy, specific drugs, and bariatric surgery. Nowadays, bariatric surgery is the most effective treatment modality in morbidly obese patients; it is superior to nonsurgical interventions and leads to improvement in medical comorbidities, such as type 2 diabetes, hypertension, dyslipidemia, and obstructive sleep apnea [2-4].

One of the longest weight-loss studies - the Swedish Obese Subjects - evaluated the long-term effects of various bariatric procedures and demonstrated a significant reduction in cardiovascular and cancer-related mortality and a significant improvement in quality of life $[5,6]$. Despite multiple benefits, a number of surgical and gastrointestinal complications can occur following bariatric procedures. The mean mortality rate is low $(0.3 \%$ for all procedures), comparable to that for laparoscopic cholecystectomy (0.3-0.6\%). Nutritional deficiencies deserve careful consideration.

The most commonly performed bariatric procedures are Roux-en-Y gastric bypass (RYGB) and laparoscopic sleeve gastrectomy (LSG) [7]. Both RYGB and LSG may lead to malnutrition [8]. LSG is associated with a smaller risk for nutritional deficiencies, while gastric bypass is as-

\section{KARGER}

(c) 2019 S. Karger AG, Basel 
sociated with increased nutritional deficiency because the procedure is more complex and changes the gastrointestinal anatomy. Recent studies comparing LSG and RYGB have proven that these types of operation may lead to a similar weight reduction effect but cause different micronutrient deficiencies [9-12].

According to the S3 Guideline published by the German Society for Metabolic \& Bariatric Surgery in 2018, patients should undergo appropriate nutritional evaluation including micronutrient measurement before and after bariatric surgery. Types of malnutrition after bariatric surgery include protein-energy malnutrition and deficiencies of micronutrients, such as iron, folate, vitamin $\mathrm{A}$, and vitamin $\mathrm{B}_{12}$. Bariatric patients who do not adhere to the recommended dietary guidelines are at a greater risk of developing relevant malnutrition [13]. Nutritional deficiencies can present with a wide range of clinical symptoms depending on the nutrients that are lacking, the extent, and the duration of deficiency. Female gender is a risk factor for pre- and postoperative deficiencies, as proposed in several studies $[14,15]$.

To date, most postoperative nutritional evaluation studies have a follow-up range between one and two years, with limited data available beyond the 2-year postLSG interval. Only few studies have investigated the nutritional status of LSG during a long-term follow-up of $>4$ years after surgery [16-18]. This article focuses on the main nutritional deficiencies related to bariatric procedures, presenting important clinical symptoms caused by hypovitaminosis or micronutrient deficits.

\section{Operative Procedures}

In the past, two different kinds of bariatric procedure were differentiated: malabsorptive and restrictive. However, today it is clear that weight loss does not result only from greatly reduced food intake, but it involves many changes in neuronal and hormonal signals projecting from and to the intestine. All these mechanisms contribute to metabolic improvement and weight loss; and today, they are still not always fully understood. The most commonly performed bariatric surgeries are shown in Figure 1.

\section{Laparoscopic Sleeve Gastrectomy}

In LSG, the stomach is divided vertically, reducing the amount of volume by $75 \%$. This is an optimal procedure for extremely obese patients and young women. The pylorus is preserved.

\section{Roux-en-Y Gastric Bypass}

Performing a RYGB means creating a small pouch with a volume of $10-20 \mathrm{~mL}$. This permits the food to by- pass most of the stomach and enter the small intestine via an anastomosis. After approximately $150 \mathrm{~cm}$, the next anastomosis is made to the biliary limb. This way, the food also bypasses the initial loop of the small intestine.

\section{Biliopancreatic Diversion with Duodenal Switch}

On the one hand, biliopancreatic diversion with duodenal switch (BPD-DS) is the bariatric procedure resulting in the greatest weight loss, but, on the other hand, it is also associated with many disadvantages because of nutritional complications. In this procedure, a distal horizontal gastrectomy is performed that leaves $200-250 \mathrm{~mL}$ of the upper stomach, which is anastomosed to the distal $250 \mathrm{~cm}$ of the small intestine. The biliopancreatic limb is then connected to the alimentary limb $50 \mathrm{~cm}$ proximal to the ileocecal valve, so that only $50 \mathrm{~cm}$ of common limb is left, resulting in a marked malabsorption of fat and protein but also of other micronutrients.

\section{Single Anastomosis with Duodenal Switch}

This operation is a modification of BPD-DS and permits food to pass the initial loop of the small intestine. The procedure consists of only one anastomosis. The small intestine is connected to the postpyloric part of the duodenum $200 \mathrm{~cm}$ proximal to the ileocecal valve. A sleeve gastrectomy is primarily performed.

\section{Laparoscopic Adjustable Gastric Banding}

Today, laparoscopic adjustable gastric banding is no longer a standard procedure. An adjustable silicone band is placed around the fundus of the stomach creating a small pouch of approximately $30 \mathrm{~mL}$. The diameter of the outlet of the pouch can be adjusted by filling the port that is connected to the band. Formerly very popular, many bands were implanted. However, dysfunction, bands which slipped, and the need to adjust the band with X-ray guidance have greatly reduced the popularity of this procedure.

\section{Nutritional Risks following Bariatric Surgery}

Nutritional deficiencies are common in obese patients before and after bariatric surgery. Therefore, aftercare of these patients is of great importance, and regular metabolic and nutritional monitoring is highly recommended, especially for malabsorptive operative procedures, but also for LSG.

Metabolic Deficiencies following Restrictive Procedures LSG and laparoscopic adjustable gastric banding are the most common restrictive operations. The gastrointestinal passage is not changed, meaning the problem of minerals, vitamins, and micronutrients is determined by the amount 
Fig. 1. Commonly performed bariatric surgeries divided in restrictive (adjustable gastric band, sleeve gastrectomy) and malabsorptive procedures (Roux-en-Y gastric bypass, mini gastric bypass, SADI-S (single anastomosis duodenojejunal bypass with sleeve gastrectomy), and biliopancreatic diversion).

\section{Restrictive procedures}

Adjustable gastric band

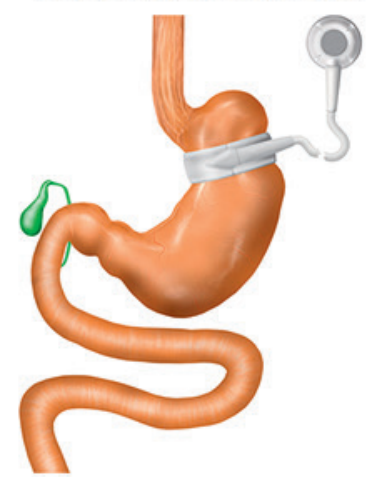

Sleeve gastrectomy

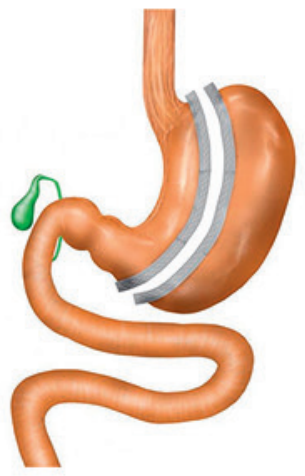

Combined restrictive and malabsorptive procedures

Roux-en-Y gastric bypass

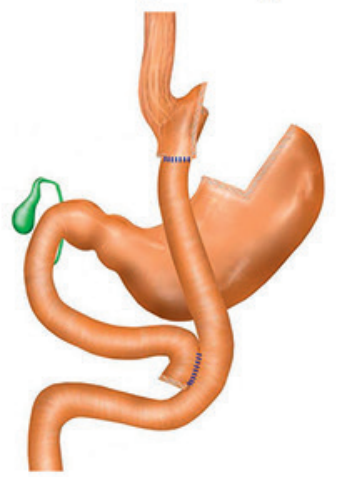

SADI-S

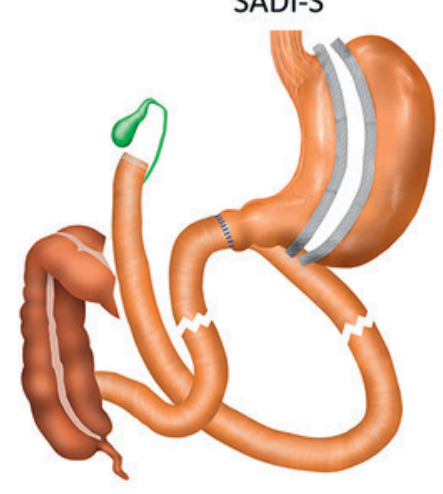

Mini gastric bypass (Omega loop)

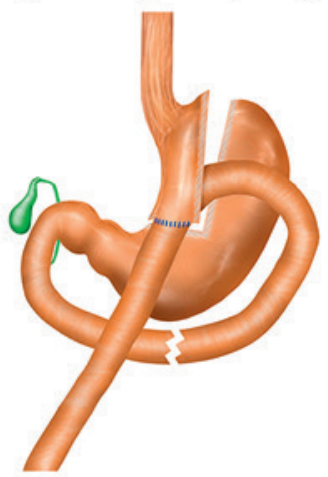

Biliopancreatic diversion

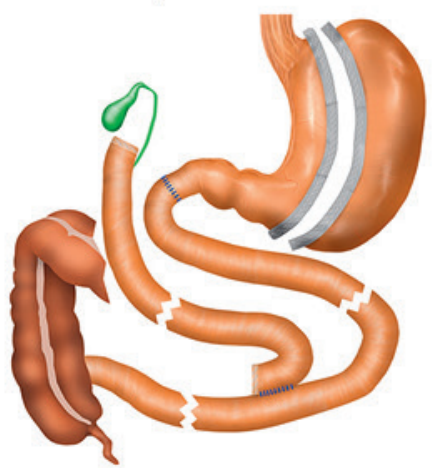

of food intake and the diversity of the various foods. Directly after the operation, the amount of food intake is very small, resulting in an insufficient intake of micronutrients. Following restrictive procedures, patients have problems especially with the $B$ vitamins [19]. Vitamins $B_{1}$ and $B_{12}$ are the most common vitamins related with neurological manifestations after bariatric surgery [20]. A vitamin $B_{1}$ deficiency can lead to beriberi, one of the most severe metabolic complications caused by an inadequate dietary intake or by persistent vomiting. If the symptoms of thiamine deficiency are recognized and treated early, symptoms disappear quickly. Therefore, early intravenous administration of thiamine is important if there is any suspicion of a thiamine deficiency with symptoms.

The literature reports the incidence of vitamin $\mathrm{B}_{12}$ deficiency to be up to $26 \%$ [9], possibly because of smaller amounts of gastric acid and the intrinsic factor [21]. Patients with neurological complications from vitamin $B$ deficiencies after LSG often have postoperative complications (marginal ulcer or stenosis of the sleeve) [20]. 
As reported by Stroh et al. [21], 24 months after LSG, permanent supplementation became necessary in 51.2\% of all patients (for iron), in $26.6 \%$ (for zinc), and in $37.8 \%$ (for a combination of calcium and cholecalciferol). In more than $30 \%$ of all patients, low serum ferritin was seen 5 years after LSG surgery, with the same rates after RYGB [10]. Iron deficiency may occur after LSG because of a change in food preferences (less meat and dairy products) and hypochlorhydria [22]. Serum ferritin measurement is best for detecting iron deficiency because it is a specific and early indicator of iron capacity. In most cases, intravenous iron administration is necessary.

\section{Metabolic Deficiencies following Malabsorptive Procedures \\ Iron}

Iron deficiency after malabsorptive procedures occurs because of maldigestion and malabsorption of iron while bypassing the duodenum and proximal jejunum and reducing gastric acid secretion. The reasons for iron deficiency are the same as those for LSG patients (changed food preferences and less intake of red meat). As a result, anemia is a common problem. Additionally, vitamin $B_{12}$ and folate deficiencies after malabsorptive procedures may aggravate anemia.

The amount of iron deficiency also depends on the malabsorptive procedure, being highest in BPD-DS (13$62 \%)$ patients. In BPD-DS patients with iron deficiency, intravenous supplementation is often necessary together with vitamin $\mathrm{C}$ administration, which permits a better intestinal uptake of iron. As prophylaxis, daily intake of additional elementary iron of 45-60 mg/day (in the case of young women $50-100 \mathrm{mg} /$ day) (insulate, fumarate, gluconate) is highly recommended [23].

\section{Vitamin $\mathrm{B}_{12}$}

Vitamin $B_{12}$ deficiency is a major cause of anemia in patients who undergo BPD-DS or RYGB. Vitamin $\mathrm{B}_{12}$ absorption takes place in the terminal ileum and requires an "intrinsic factor" that is produced in the gastric corpus. The rate of vitamin $B_{12}$ deficiency varies in different studies. Additionally, the use of proton pump inhibitors and metformin may interfere with vitamin $\mathrm{B}_{12}$ absorption. Slater et al. [24] showed no differences in $B_{12}$ deficiency between patients after RYGB and those after BPD-DS.

Clinical symptoms of vitamin $B_{12}$ deficiency are anemia and neurological complications, such as ataxia, paresthesia, muscle weakness, and decreased reflexes. Oral or, even better, intramuscular supplementation of vita$\min B_{12}$ is recommended because, at only $1-5 \%$, intestinal absorption is very low. Administration of 1,000-3,000 $\mu \mathrm{g}$ every three to six months is recommended in the S3 Guideline. Even though there is only little evidence after restrictive procedures, administration is recommended after all bariatric procedures except laparoscopic banding [23].

\section{Vitamin $B_{1}$}

Thiamine (vitamin $B_{1}$ ) deficiency occurs in about $18 \%$ of all patients after bariatric surgery. It is associated with different symptoms while affecting different organs (heart, gastrointestinal tract, peripheral and central nervous system). If thiamine deficiency is detected prior to surgery, supplementation is crucial because postoperative prolonged vomiting, reduced absorption, and an altered diet may worsen the deficiency. Clinical symptoms of thiamine deficiency must be treated early; otherwise, permanent neurological deficits may occur. There are three different forms: "wet" beriberi (cardiac symptoms), "dry" beriberi (most common type, symptoms include peripheral neuropathy), and cerebral beriberi (Wernicke encephalopathy).

Cerebral beriberi is associated with acute and severe thiamine deficiency, and it affects the spinal cord and the central nervous system. Symptoms are severe cognitive and psychotic disorders, confusion, gait ataxia, and dysfunction of cranial nerves [21]. Risk factors are rapid weight loss and long-term parenteral nutrition [25]. Following cerebral beriberi, most patients have residual neurological deficiency. A daily dose of $1.1 \mathrm{mg}$ for females and $1.2-1.5 \mathrm{mg}$ for males is essential to prevent thiamine deficiency.

Treatment of thiamine-associated neurological disorders with $100 \mathrm{mg}$ of thiamine administered intravenously or intramuscularly is required and should be combined with magnesium and other $\mathrm{B}$ complex vitamins. Female gender and higher age are two risk factors for the development of cerebral beriberi [26].

\section{Fat-Soluble Vitamins}

Fat-soluble vitamins may be low after any malabsorptive surgical procedure. Malabsorption of fat-soluble vitamins develops as a result of fat malabsorption in the distal ileum and affects vitamins A, E, K, and D. Vitamin A deficiency occurs in $69 \%$ of all patients at 4 years after malabsorptive surgery and is treated by supplementing 5,000-10,000 IU/day. Vitamin E deficiency is rare following bariatric surgery. The incidence ranges from 2 to $5 \%$ between 2 and 4 years after surgery and mostly after BPDDS patients. Vitamin K deficiency is merely a laboratory diagnosis. There are no patients with clinical signs of deficiency (prolonged bleeding, early bruising, and hemorrhage) [19].

\section{Vitamin D}

Changes in bone metabolism are a common long-term complication following bariatric surgery. Calcium and vitamin D deficiencies are the two most important factors 
of accelerated bone loss after bariatric surgery. Calcium and vitamin $\mathrm{D}$ deficiencies often preexist before bariatric surgery or occur very early after weight loss following bariatric surgery, especially after bypass operations. Vitamin $\mathrm{D}$ is reabsorbed in the jejunum and ileum, and calcium is reabsorbed in the proximal jejunum.

Vitamin D deficiency in obese patients may be caused by less exposure to sunlight, less vitamin D consumption, and sequestration of vitamin $D$ in adipose tissue [27]. For prophylaxis, amounts of 3,000 IU/day are recommended. A preexisting vitamin $\mathrm{D}$ deficiency should be treated before surgery and is very common (between 25 and 80\%).

Calcium deficiencies are caused by a reduction in calcium absorption as a result of bypassing the duodenum and proximal jejunum. Often calcium intake is also low because of an intolerance to milk products after surgery. Parathyroid hormone levels are often high before and after bariatric surgery because calcium is mobilized out of the bone resulting in a higher risk of osteoporosis and fractures [28]. The amount of vitamin D supplementation varies according to the bariatric surgery that is performed (highest doses after BPD-DS) and the studies which are cited, but 3,000 IU/day is the minimum.

\section{Zinc and Copper}

Zinc resorption correlates with fat absorption. Zinc deficiency is highest in patients after BPD-DS (up to $70 \%$ ). If a malabsorptive procedure is planned, preoperative screening is mandatory. Following RYGB or BPDDS, patients should be screened annually. Daily supplementation of $8-15 \mathrm{mg}$ is recommended.

Copper reabsorption is lower if the amount of gastric acid is reduced. If a daily dose of $2 \mathrm{mg}$ is administered, there is no need for laboratory monitoring.

\section{Magnesium}

Magnesium deficiency also plays a role in the development of osteoporosis following bariatric surgery. It should be supplemented as magnesium citrate $(300 \mathrm{mg} /$ day). Laboratory monitoring is not necessary.

\section{Protein Malnutrition}

Protein malnutrition is one of the most severe macronutrient problems encountered after malabsorptive surgery. It can occur after RYGB or even after LSG, but it most commonly affects patients after BPD-DS because of poor protein digestion and absorption as a consequence of changed biliary and pancreatic function [29]. In RYGB or LSG patients, it occurs because protein food sources are avoided and long vomiting phases occur. We recommend to all our bariatric patients that they keep an eye on their daily protein dose and that if the recommended daily protein intake of $60-120 \mathrm{~g}(1.5 \mathrm{~g} / \mathrm{kg}$ ideal body weight) is not reached, they should take protein shakes, protein powder, or protein water. Clinical signs of protein malnutrition include edema, muscle loss, increasing appetite, and skin, hair, and nail problems. The amount should be increased by $30 \%$ for patients after BPD-DS. Exercise is also an important factor in maintaining muscle mass.

\section{"Sarcopenic Obesity"}

Sarcopenic obesity is defined as obesity in combination with sarcopenia that occurs in older individuals, in those with chronic obstructive pulmonary disease, diabetes type II, in obese patients with malignant disorders, or in obese patients with other consuming diseases. It is associated with negative health outcomes. Excess energy intake, physical inactivity, low-grade inflammation, insulin resistance, and changes in the hormonal milieu maylead to the development of so-called sarcopenic obesity. Originally, it was believed that the culprit of agerelated muscle weakness was a reduction in muscle mass, but it is now clear that changes in muscle composition and quality are predominant [30]. Also, sarcopenic obesity may occur in patients undergoing conservative weight loss therapy or bariatric surgery when the protein intake is not enough.

Dual energy X-ray absorptiometry might be the most accurate method for body composition measurement in obese patients. Selected CT scan is an alternative for the measurement of muscle mass [31]. The European Working Group on Sarcopenia in Older People suggests two different methods to assess muscle mass: dual energy Xray absorptiometry and bio-electrical impedance analysis [32]. The latter is a simple, quick, and non-invasive method, but it might overestimate fat-free mass in obese subjects [33]. There is a lack in normal ranges for the fat-free mass index (fat-free mass/height ${ }^{2}$ ) and for obese individuals, too. There are no commonly accepted criteria for sarcopenic obesity beyond those for sarcopenia and obesity separately. The risk of skeletal muscle loss and dysfunction should be considered in obese patients, particularly in the presence of advanced age ( $>65$ years) or in obese patients with metabolic complications. Efforts should be made to monitor skeletal muscle function and mass and to prevent or minimize their loss in patients undergoing bariatric surgery procedures, particularly with regard to malabsorptive ones, and in those undergoing hypocaloric dietary treatment, especially in the presence of advanced age and/or comorbidities [34]. It may also concern patients after long immobilization as in the intensive care unit [35], as well as patients suffering from specific endocrine disorders.

In obese patients with clinical evidence of loss of skeletal muscle function and mass or in those with at-risk conditions (metabolic complications, chronic or acute diseases) undergoing weight-loss programs, treatment 
Table 1. Timetable and scope of routine laboratory measurement for nutrition monitoring after bariatric surgery

\begin{tabular}{llll}
\hline & LSG $^{\mathrm{a}}$ & RYGB $^{\mathrm{b}}$ & BPD-DS $^{\mathrm{b}}$ \\
\hline Protein & $\mathrm{x}$ & $\mathrm{x}$ & $\mathrm{x}$ \\
Ferritin & $\mathrm{x}$ & $\mathrm{x}$ & $\mathrm{x}$ \\
Vitamin $\mathrm{B}_{12}$ & $\mathrm{x}$ & $\mathrm{x}$ & $\mathrm{x}$ \\
Folate & $\mathrm{x}$ & $\mathrm{x}$ & $\mathrm{x}$ \\
Thiamine & $\mathrm{x}$ & $\mathrm{x}$ & $\mathrm{x}$ \\
Vitamin D & $\mathrm{x}$ & $\mathrm{x}$ & $\mathrm{x}$ \\
Calcium & $\mathrm{x}$ & $\mathrm{x}$ & $\mathrm{x}$ \\
\hline Vitamin E & & $\mathrm{x}$ & $\mathrm{x}$ \\
Vitamin K & & $\mathrm{x}$ & $\mathrm{x}$ \\
Copper & & & $\mathrm{x}$ \\
Parathyroid hormone & & $\mathrm{x}$ & $\mathrm{x}$ \\
Vitamin A & & $\mathrm{x}$ & $\mathrm{x}$ \\
Selenium & & $\mathrm{x}$ & $\mathrm{x}$ \\
Zinc & & $\mathrm{x}$ \\
\hline
\end{tabular}

Based on recommendations from the S3 Guideline $[19,23,40$ 42]. LSG, laparoscopic sleeve gastrectomy; RYGB, Roux-en-Y gastric bypass; BPD-DS, biliopancreatic diversion with duodenal switch.

a After 6 and 12 months, then yearly.

b After 3, 6, and 12 months, then yearly.

should be associated with precaution measurements to prevent, limit, or treat skeletal muscle alterations. Approaches should include nutritional care and physical exercise. Adequate protein intake of $1 \mathrm{~g} / \mathrm{kg}$ ideal body weight per day should be provided in healthy non-geriatric individuals. In addition, higher protein intakes are increasingly recommended by guidelines and expert groups for high-risk patients. Obese intensive care unit patients undergoing acute metabolic stress with likely substantial muscle loss and weakness have been specifically addressed in recent guidelines with recommendations of very high protein intakes of up to $2.2 \mathrm{~g} / \mathrm{kg} /$ day through medical nutritional support [36].

It was shown in a single-center study that sarcopenia did not impact bariatric surgery outcomes 1 year after RYGB or LSG. Early complication rates were not significantly different between the sarcopenia and the no-sarcopenia group [37]. Sarcopenic obese patients are not at a higher operative risk, as the early complication rates and the duration of the surgical procedure were similar in both groups. In conclusion, it seems that weight loss is still beneficial, independent of the skeletal muscle mass, to improve high altitude-induced systemic hypertension, hypertension, type 2 diabetes, dyslipidemia, and obstructive sleep apnea syndrome. On the other hand, studies have shown that sarcopenic obesity in elderly people is associated with higher mortality risk in comparison to obesity or sarcopenia alone [38, 39].

\section{Preoperative Nutrition State}

Obese patients often have preexisting micronutrient deficiencies because of their diet, unhealthy eating behavior, and low sun exposure. Therefore, early preoperative micronutrient status and treatment of deficiencies are recommended, especially for malabsorptive operative procedures. Life-long nutritional monitoring is mandatory after bariatric surgery. Following bariatric surgery, all patients should be monitored at the center where they were operated. The routine monitoring recommendation in the S3 Guideline is shown in Table 1.

\section{Conclusion}

Bariatric surgery is very effective in treating obese patients and shows good weight-loss results. These operations also improve associated comorbidities. On the other hand, nutritional deficiencies are very common after and even before bariatric surgery. Therefore, following bariatric surgery, obese patients require life-long postoperative clinical and laboratory monitoring to diagnose deficiencies of vitamins, trace elements, and minerals and to correct them with supplements. Following bariatric surgery, patients should undergo a life-long follow-up by experts in this field because serious malnutrition or deficiencies can cause severe, even life-threatening, problems.

In Germany, the hospital where the bariatric surgery was performed is responsible for aftercare. Unfortunately, no standardized aftercare regimes exist for these patients, and the costs for nutritional supplements are paid by the patients themselves.

\section{Disclosure Statement}

The authors certify that they have no commercial associations that might pose a conflict of interest in connection with the submitted article.

\section{Funding Sources}

There are no funding sources supporting the work. All institutional or corporate affiliations are acknowledged in the article.

\section{Author Contributions}

Jessica Lange wrote the manuscript with support from Alfred Königsrainer. 
1 WHO Global InfoBase [accessed 2014 Jan 27]. Available from: https://apps.who.int/ infobase.

2 Aminian A, Brethauer SA, Andalib A, Punchai S, Mackey J, Rodriguez J, et al. Can Sleeve Gastrectomy "Cure" Diabetes? Long-term Metabolic Effects of Sleeve Gastrectomy in Patients With Type 2 Diabetes. Ann Surg. 2016 Oct;264(4):674-81.

3 Aminian A, Zelisko A, Kirwan JP, Brethauer SA, Schauer PR. Exploring the impact of bariatric surgery on high density lipoprotein. Surg Obes Relat Dis. 2015 Jan-Feb;11(1):23847.

4 Aminian A, Daigle CR, Romero-Talamás $\mathrm{H}$, Kashyap SR, Kirwan JP, Brethauer SA, et al. Risk prediction of complications of metabolic syndrome before and 6 years after gastric bypass. Surg Obes Relat Dis. 2014 Jul-Aug; 10(4): $576-82$.

5 Sjöström L, Narbro K, Sjöström CD, Karason K, Larsson B, Wedel H, et al.; Swedish Obese Subjects Study. Effects of bariatric surgery on mortality in Swedish obese subjects. N Engl J Med. 2007 Aug;357(8):741-52.

6 Sjöström L, Peltonen M, Jacobson P, Sjöström $\mathrm{CD}$, Karason K, Wedel H, et al. Bariatric surgery and long-term cardiovascular events. JAMA. 2012 Jan;307(1):56-65.

7 Ponce J, Nguyen NT, Hutter M, Sudan R, Morton JM. American Society for Metabolic and Bariatric Surgery estimation of bariatric surgery procedures in the United States, 2011-2014. Surg Obes Relat Dis. 2015 NovDec;11(6):1199-200.

8 Allied Health Sciences Section Ad Hoc Nutrition Committee, Aills L, Blankenship J, Buffington C, Furtado M, Parrott J. ASMBS Allied Health Nutritional Guidelines for the Surgical Weight Loss Patient. Surg Obes Relat Dis. 2008;4(5):S73-108.

9 Gehrer S, Kern B, Peters T, Christoffel-Courtin C, Peterli R. Fewer nutrient deficiencies after laparoscopic sleeve gastrectomy (LSG) than after laparoscopic Roux-Y-gastric bypass (LRYGB)-a prospective study. Obes Surg. 2010 Apr;20(4):447-53.

10 Alexandrou A, Armeni E, Kouskouni E, Tsoka E, Diamantis T, Lambrinoudaki I. Crosssectional long-term micronutrient deficiencies after sleeve gastrectomy versus Roux-enY gastric bypass: a pilot study. Surg Obes Relat Dis. 2014 Mar-Apr;10(2):262-8.

11 Coupaye M, Rivière $\mathrm{P}$, Breuil MC, Castel B, Bogard C, Dupré T, et al. Comparison of nutritional status during the first year after sleeve gastrectomy and Roux-en- $Y$ gastric bypass. Obes Surg. $2014 \mathrm{Feb}$;24(2):276-83.

12 Antoniewicz A, Kalinowski P, Kotulecka KJ, Kocoń P, Paluszkiewicz R, Remiszewski P, et al. Nutritional Deficiencies in Patients after Roux-en-Y Gastric Bypass and Sleeve Gastrectomy during 12-Month Follow-Up. Obes Surg. 2019 Jun. https://doi.org/10.1007/ s11695-019-03985-3.

13 Beckman L, Earthman C. Nutritional implications of bariatric surgery and the role of registered dietitians. J Acad Nutr Diet. 2013 Mar; 113(3):398-9.
14 Ben-Porat T, Elazary R, Yuval JB, Wieder A, Khalaileh A, Weiss R. Nutritional deficiencies after sleeve gastrectomy: can they be predicted preoperatively? Surg Obes Relat Dis. 2015 Sep-Oct;11(5):1029-36.

15 Schweiger C, Weiss R, Berry E, Keidar A. Nutritional deficiencies in bariatric surgery candidates. Obes Surg. 2010 Feb;20(2):193-7.

16 Saif T, Strain GW, Dakin G, Gagner M, Costa R, Pomp A. Evaluation of nutrient status after laparoscopic sleeve gastrectomy 1, 3, and 5 years after surgery. Surg Obes Relat Dis. 2012 Sep-Oct;8(5):542-7.

17 Gillon S, Jeanes YM, Andersen JR, Våge V. Micronutrient Status in Morbidly Obese Patients Prior to Laparoscopic Sleeve Gastrectomy and Micronutrient Changes 5 years Post-surgery. Obes Surg. 2017 Mar;27(3): 606-12.

18 Moizé V, Andreu A, Flores L, Torres F, Ibarzabal A, Delgado S, et al. Long-term dietary intake and nutritional deficiencies following sleeve gastrectomy or Roux-En-Y gastric bypass in a mediterranean population. J Acad Nutr Diet. 2013 Mar;113(3):400-10.

19 Stroh C, Benedix F, Meyer F, Manger T. [Nutrient Deficiencies after Bariatric Surgery Systematic Literature Review and Suggestions for Diagnostics and Treatment]. Zentralbl Chir. 2015 Aug;140(4):407-16.

20 Punchai S, Hanipah ZN, Meister KM, Schauer PR, Brethauer SA, Aminian A. Neurologic Manifestations of Vitamin B Deficiency after Bariatric Surgery. Obes Surg. 2017 Aug;27(8): 2079-82.

21 Stroh C, Manger T, Benedix F. Metabolic surgery and nutritional deficiencies. Minerva Chir. 2017 Oct;72(5):432-41.

22 Lupoli R, Lembo E, Saldalamacchia G, Avola CK, Angrisani L, Capaldo B. Bariatric surgery and long-term nutritional issues. World J Diabetes. 2017 Nov;8(11):464-74.

23 Handzlik-Orlik G, Holecki M, Orlik B, Wyleżoł M, Duława J. Nutrition management of the post-bariatric surgery patient. Nutr Clin Pract. 2015 Jun;30(3):383-92.

24 Slater GH, Ren CJ, Siegel N, Williams T, Barr D, Wolfe B, et al. Serum fat-soluble vitamin deficiency and abnormal calcium metabolism after malabsorptive bariatric surgery. J Gastrointest Surg. 2004 Jan;8(1):48-55.

25 Kröll D, Laimer M, Borbély YM, Laederach K, Candinas D, Nett PC. Wernicke Encephalopathy: a Future Problem Even After Sleeve Gastrectomy? A Systematic Literature Review. Obes Surg. 2016 Jan;26(1):205-12.

26 Stroh C, Meyer F, Manger T. Beriberi, a severe complication after metabolic surgery - review of the literature. Obes Facts. 2014;7(4):24652.

27 Dogan K, Homan J, Aarts EO, de Boer H, van Laarhoven CJ, Berends FJ. Long-term nutritional status in patients following Roux-en-Y gastric bypass surgery. Clin Nutr. 2018 Apr; 37(2):612-7.

28 Nakamura KM, Haglind EG, Clowes JA, Achenbach SJ, Atkinson EJ, Melton LJ 3rd, et al. Fracture risk following bariatric surgery: a population-based study. Osteoporos Int. 2014 Jan;25(1):151-8.
29 Faintuch J, Matsuda M, Cruz ME, Silva MM, Teivelis MP, Garrido AB Jr, et al. Severe protein-calorie malnutrition after bariatric procedures. Obes Surg. 2004 Feb;14(2):175-81.

30 Stenholm S, Harris TB, Rantanen T, Visser M, Kritchevsky SB, Ferrucci L. Sarcopenic obesity: definition, cause and consequences. Curr Opin Clin Nutr Metab Care. 2008 Nov;11(6): 693-700.

31 Lemos T, Gallagher D. Current body composition measurement techniques. Curr Opin Endocrinol Diabetes Obes. 2017 Oct;24(5): 310-4.

32 Choi KM. Sarcopenia and sarcopenic obesity. Endocrinol Metab (Seoul). 2013 Jun;28(2): 86-9.

33 Faria SL, Faria OP, Cardeal MD, Ito MK. Validation study of multi-frequency bioelectrical impedance with dual-energy X-ray absorptiometry among obese patients. Obes Surg. 2014 Sep;24(9):1476-80.

34 Barazzoni R, Bischoff SC, Boirie Y, Busetto L, Cederholm T, Dicker D, et al. Sarcopenic obesity: time to meet the challenge. Clin Nutr. 2018 Dec;37(6 6 Pt A):1787-93.

35 Puthucheary ZA, Rawal J, McPhail M, Connolly B, Ratnayake G, Chan P, et al. Acute skeletal muscle wasting in critical illness. JAMA. 2013 Oct;310(15):1591-600.

36 Bauer J, Biolo G, Cederholm T, Cesari M, Cruz-Jentoft AJ, Morley JE, et al. Evidencebased recommendations for optimal dietary protein intake in older people: a position paper from the PROT-AGE Study Group. J Am Med Dir Assoc. 2013 Aug;14(8):542-59.

37 Mastino D, Robert M, Betry C, Laville M, Gouillat C, Disse E. Bariatric Surgery Outcomes in Sarcopenic Obesity. Obes Surg. 2016 Oct;26(10):2355-62.

38 Batsis JA, Mackenzie TA, Barre LK, LopezJimenez F, Bartels SJ. Sarcopenia, sarcopenic obesity and mortality in older adults: results from the National Health and Nutrition Examination Survey III. Eur J Clin Nutr. 2014 Sep;68(9):1001-7.

39 Atkins JL, Whincup PH, Morris RW, Lennon LT, Papacosta O, Wannamethee SG. Sarcopenic obesity and risk of cardiovascular disease and mortality: a population-based cohort study of older men. J Am Geriatr Soc. 2014 Feb;62(2):253-60.

40 Heber D, Greenway FL, Kaplan LM, Livingston E, Salvador J, Still C; Endocrine Society. Endocrine and nutritional management of the post-bariatric surgery patient: an Endocrine Society Clinical Practice Guideline. J Clin Endocrinol Metab. 2010 Nov;95(11): 4823-43.

41 Billeter AT, Fischer L, Wekerle AL, Senft J, Müller-Stich B. Malabsorption as a Therapeutic Approach in Bariatric Surgery. Viszeralmedizin. 2014 Jun;30(3):198-204.

42 Stein J, Stier C, Raab H, Weiner R. Review article: the nutritional and pharmacological consequences of obesity surgery. Aliment Pharmacol Ther. 2014 Sep;40(6):582-609. 